STRUCTURAL SCIENCE CRYSTAL ENGINEERING MATERIALS

ISSN 2052-5206
Keywords: book review; structural inorganic chemistry
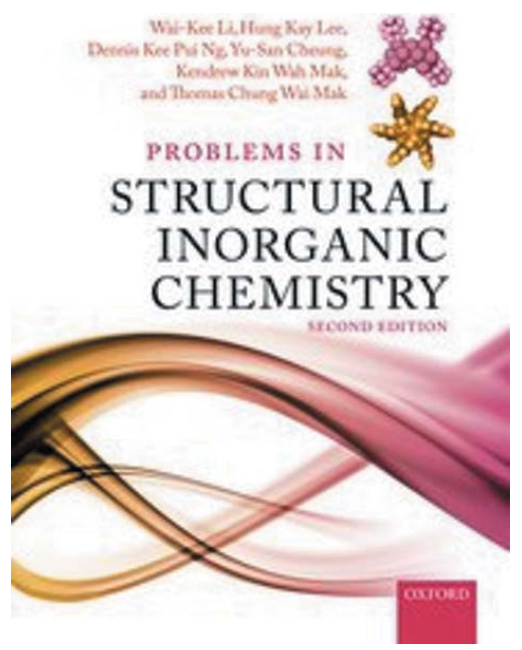

(C) 2019 International Union of Crystallography

\section{Problems in Structural Inorganic Chemistry. Second edition. By Wai-Kee Li, Hung Kay Lee, Dennis Kee Pui Ng, Yu-San Cheung, Kendrew Kin Wah Mak, and Thomas Chung Wai Mak. Oxford University Press, 2018, Hardcover, pp. 480. Price GBP 75.00. ISBN 9780198823902}

\author{
Michele Catti*
}

Dipartimento di Scienza dei Materiali, Università degli Studi di Milano Bicocca, Via Roberto Cozzi, 55, Milano, Italy. *Correspondence e-mail: michele.catti@unimib.it

This book was conceived as a problem text for Part I (Fundamentals of Chemical Bonding) and Part II (Symmetry in Chemistry) of Advanced Structural Inorganic Chemistry, Oxford University Press, 2008, written by the same authors. The second edition of Problems in Structural Inorganic Chemistry is organized into 13 chapters, each of which contains a Problems section followed by corresponding Solutions section. Problems were selected carefully and presented in a clear way. According to the authors, senior undergraduate students of chemistry should be the target of the book; however, several problems may be challenging also for graduate people who are not specialists in the subject. Thus, in my opinion the sample of possibly interested readers is quite broad, reaching also $\mathrm{PhD}$ students who want to deepen their understanding of subsidiary subjects of their thesis. The Solutions sections are particularly valuable, as each case is explained extensively, contributing to the insight of the reader into the specific issue.

Basic topics of quantum chemistry and chemical bond theory are covered in the first nine chapters, which were already present in the first edition of the book (2012). Many of the concerned problems are similar to those currently attached to physical chemistry courses for undergraduate students. Yet examples are selected with great variety from a rich knowledge of chemistry. In particular, Chapters 5 (Molecular Symmetry) and 6 (Molecular Geometry and Bonding) are most relevant for students inclined to chemical crystallography, although only intramolecular structure is accounted for in the problems.

The four new chapters cover subjects more directly pertaining to crystal and intermolecular structure, where the general abilities acquired by problem-solving in the previous chapters can be applied. Chapter 10, entitled Crystal Structure, contains a comprehensive selections of 47 problems on space group symmetry, systematic absences, bond distance calculations, and other classic crystallographic topics. The problems of the last chapters are more specific to real inorganic or organometallic chemistry (the subjects are transition metal chemistry, metal clusters: bonding and reactivity, bioinorganic chemistry). Most items presented in problems are useful complements and examples for understanding the general principles dealt with in Advanced Structural Inorganic Chemistry.

In summary, this text can be a valuable aid not only for students but also for lecturers, who could be inspired by it to design new problems and/or discussion themes for their classes. 